\title{
Ibuprofen-loaded poly(lactic-co-glycolic acid) films for controlled drug release
}

\author{
This article was published in the following Dove Press journal: \\ International Journal of Nanomedicine \\ 3I March 20II \\ Number of times this article has been viewed
}

\section{Jianmei Pang' \\ Yuxia Luan' \\ Feifei $\mathrm{Li}^{\prime}$ \\ Xiaoqing $\mathrm{Cai}^{\prime}$ \\ Jimin $\mathrm{Du}^{2}$ \\ Zhonghao $\mathrm{Li}^{3}$}

'School of Pharmaceutical Science, Shandong University, Jinan, Shandong Province, PR China; ${ }^{2}$ School of Chemistry and Chemical Engineering, Anyang Normal University, Henan Province, PR China; ${ }^{3}$ School of Materials Science and Engineering, Shandong University, Jinan, Shandong Province, PR China
Correspondence: Yuxia Luan School of Pharmaceutical Science, Shandong University, Jinan, Shandong Province, 250012, PR China

Tel $+8653 \mid 88382007$

Fax $+8653 \mid 8838273$ I

Email yuxialuan@sdu.edu.cn
Abstract: Ibuprofen- (IBU) loaded biocompatible poly(lactic-co-glycolic acid) (PLGA) films were prepared by spreading polymer/ibuprofen solution on the nonsolvent surface. By controlling the weight ratio of drug and polymer, different drug loading polymer films can be obtained. The synthesized ibuprofen-loaded PLGA films were characterized with scanning electron microscopy, powder X-ray diffraction, and differential scanning calorimetry. The drug release behavior of the as-prepared IBU-loaded PLGA films was studied to reveal their potential application in drug delivery systems. The results show the feasibility of the as-obtained films for controlling drug release. Furthermore, the drug release rate of the film could be controlled by the drug loading content and the release medium. The development of a biodegradable ibuprofen system, based on films, should be of great interest in drug delivery systems.

Keywords: ibuprofen, controlled release, poly(lactic-co-glycolic acid), films

\section{Introduction}

Nowadays, poly(lactic-co-glycolic acid) (PLGA) is widely applied in controlled drug delivery systems due to its biodegradability, toxicological safety, and good biocompatibility. ${ }^{1-5}$ Specifically, PLGA-based carriers have been used in long-term drug delivery systems because they have the potential to control drug release from a few days up to several months. ${ }^{6,7}$ In general, PLGA can degrade into water soluble, non-toxic products of normal metabolism through hydrolysis which is important for its practical application. ${ }^{8}$ Moreover, PLGA is one of the few synthetic polymers which have been approved for human clinical use. Several products such as Lupron Depot based on PLGA microparticles are available on the market. ${ }^{5,9}$ However, PLGAbased carriers are mostly solid particles including nanoparticles, microparticles, and microcapsules, which have the disadvantages of low drug loading capacity, easy aggregation, and polydispersable particle sizes..$^{10}$ Therefore, it is necessary to prepare aspherical carriers instead of particles. Various biocompatible and biodegradable polymer films have been widely used for controlled drug release due to their excellent characteristics, such as high surface area, softness, absorbency, and ease of fabrication into many product forms. ${ }^{11-16}$ Until now, a number of methods have been developed for fabrication of polymer films, such as layer-by-layer (LBL) method ${ }^{17}$ and solvent evaporation technique. ${ }^{18}$ However, the reported methods usually involve some cumbersome processing steps and are time-consuming. Therefore developing a simple route to prepare drug-loaded fibers is still a great challenge.

Ibuprofen (IBU) is classified by the Biopharmaceutics Classification System (BCS) as a Class II active pharmaceutical ingredient as it presents low solubility and 
high permeability. ${ }^{19}$ Thus, increasing the solubility of IBU to enhance its bioavailability is the major obstacle..$^{20,21}$ Previous studies indicate that the formulated IBU microspheres have a high initial burst resulting from the accumulation of drug crystals on the surface of microspheres. ${ }^{8,22}$ In some cases, the formulated IBU microspheres are unsuitable for well controlled release.

In this paper we present a very simple and general route to prepare drug-loaded films. ${ }^{23,24}$ The biocompatible PLGA and IBU are used as the model polymer and drug, respectively. Both PLGA and IBU are dissolved in tetrahydrofuran (THF), and then $50 \mu \mathrm{L}$ of the solution is injected on the surface of the water. PLGA and IBU are indissoluble in water while THF and water are mixable. Thus, the IBU-loaded PLGA film is obtained on the surface of water. Films with different drug-loading contents could be prepared by controlling the ratios of IBU and PLGA. The aims of this study are to prepare IBU-loaded PLGA films with a simple route, and to characterize these films for their potential applications in drug delivery systems.

\section{Materials and methods Materials}

L-lactic acid (LA) and glycolic acid (GA) were purchased from Sinopharm Chemical Reagent Co. Ltd (Shanghai, China). PLGA was synthesized according to the literature. ${ }^{25}$ The $M_{\mathrm{W}}$ of PLGA $\left(\mathrm{LA} / \mathrm{GA}=75 / 25, M_{\mathrm{W}}=80,000 \mathrm{~g} / \mathrm{mol}\right)$ was determined by the inherent viscosity. IBU was bought from Aldrich. Double-distilled water was used in all the experiments. All chemicals were used as received.

\section{Preparation of drug-loaded PLGA films}

For a typical synthesis, appropriate amounts of PLGA and IBU were dissolved in tetrahydrofuran (THF). The weight ratio of THF/PLGA/IBU in the solution was $W_{\mathrm{THF}} / W_{\mathrm{PLGA}}$ ' $W_{\mathrm{IBU}}=85 / 12.5 / 2.5$. A pipette was used to take $50 \mu \mathrm{L}$ of the solution and then the solution was injected on the surface of distilled water in a beaker (the distance between the water surface and the drop source was $1 \mathrm{~cm}$ ). The solution spread quickly on the surface of water and solidified into a film within 3 seconds then the film was taken out at once and washed with distilled water and dried in air at $40^{\circ} \mathrm{C}$. For the samples synthesized with different weight ratios, such as $W_{\mathrm{THF}} / W_{\text {PLGA }}$ ' $W_{\mathrm{IBU}}=85 / 11.25 / 3.75$ and $W_{\mathrm{THF}} / W_{\mathrm{PLGA}} / W_{\mathrm{IBU}}=85 / 10 / 5$, the synthetic procedures were similar to the above.

\section{Drug loading and encapsulation efficiency}

A certain amount of dried film was dissolved in $10 \mathrm{~mL}$ of THF. The UV absorbance of the solution was measured using a UV spectrophotometer at wavelength $264 \mathrm{~nm}$ which was a typical absorbance of IBU in THF. Pure THF was used for a blank experiment before the UV measurement. Drug loading content (DLC) and encapsulation efficiency (EE) were determined using the following equations.

$$
\begin{gathered}
\operatorname{DLC}(\%)=(\text { IBU weight in film } / \text { film weight }) \times 100 \% \\
\mathrm{EE}(\%)=\left(\begin{array}{c}
\text { actual weight of IBU } / \text { theoretical } \\
\text { weight of IBU }) \times 100 \%
\end{array}\right.
\end{gathered}
$$

\section{In vitro release studies}

Three kinds of release mediums (phosphate buffered solution, PBS, $\mathrm{pH}=6.8$; phosphate buffered solution, $\mathrm{PBS}, \mathrm{pH}=7.4$; and $\mathrm{HCl}$ aqueous solution, $\mathrm{pH}=1.2$ ) were used to study the in vitro release. In drug release studies, PBS was the typical medium to mimic intestinal fluid. The release of samples with different ratios $\left(W_{\mathrm{THF}} / W_{\mathrm{PLGA}} / W_{\mathrm{IBU}}=85 / 12.5 / 2.5, W_{\mathrm{THF}} / W_{\mathrm{PLGA}} /\right.$ $W_{\mathrm{IBU}}=85 / 11.25 / 3.75$, and $\left.W_{\mathrm{THF}} / W_{\mathrm{PLGA}} / W_{\mathrm{IBU}}=85 / 10 / 5\right)$ were performed in $50 \mathrm{~mL}$ PBS ( $\mathrm{pH} 6.8)$. The influence of the release medium on the sample $\left(W_{\mathrm{THF}} / W_{\mathrm{PLGA}} / W_{\mathrm{IBU}}=85 / 12.5 / 2.5\right) \mathrm{was}$ performed in $50 \mathrm{~mL} \mathrm{HCl}$ solution $(\mathrm{pH} 1.2)$ and $\mathrm{PBS}(\mathrm{pH}$ 7.4), respectively. The in vitro experiments were carried out in a heated bath at $37^{\circ} \mathrm{C}$ in triplicate. The sample was sealed in a dialysis bag (from Solarbio, Beijing, China), which was suspended in $50 \mathrm{~mL}$ release medium in a sealed beaker under continuous stirring at a rate of $100 \mathrm{rpm} .^{26,27}$ At predetermined time intervals, $1.5 \mathrm{~mL}$ of the resultant release medium was sampled for analysis, then $1.5 \mathrm{~mL}$ of fresh release medium was immediately added to maintain the original volume. Based on the standard curve, the IBU concentration in the sampled release medium was obtained through UV experiment $(\lambda=222 \mathrm{~nm})$.

The cumulative amount of IBU released from the samples was calculated using the equation:

Cumulative amount released $(\%)=M_{\mathrm{t}} / M_{\text {total }} \times 100 \%$

$M_{\mathrm{t}}$ was the amount of IBU released from the PLGA films at time $t$ and $M_{\text {total }}$ was the total amount of IBU loaded in the PLGA films.

\section{Drug release kinetics}

The kinetics of IBU release from the films were determined by fitting different curves to distinct models. The models included Zero order, First order, Higuchi release model, Ritger-Peppas, and Biexponential model. The linear form of each function was evaluated using $R$ regression analysis.

\section{SEM}

Scanning electron microscopy (SEM) was done using a Hitachi SU-70 FESEM scanning electron microscope 
operated at $3 \mathrm{kV}$. Samples were sputtered with Au prior to imaging (the thickness of Au was $5 \mathrm{~nm}$ ).

\section{X-ray diffraction (XRD) measurements}

XRD measurements were obtained on a Rigaku Dmax-rc $\mathrm{X}$-ray diffractometer with $\mathrm{Ni}$ filtered $\mathrm{Cu} \mathrm{K} \alpha$ radiation. The angular range was from $10^{\circ}$ to $60^{\circ}$.

\section{DSC measurements}

Differential scanning calorimetry (DSC) measurements were performed using CDR-4P (Shanghai Precision and Scientific Instrument Co., Ltd. China). Weighed samples of $5 \mathrm{mg}$ were placed in aluminum pans and the samples were scanned from $40^{\circ} \mathrm{C}$ to $200^{\circ} \mathrm{C}$ using a heating rate of $10^{\circ} \mathrm{C} / \mathrm{min}$.

\section{Results and discussion}

\section{Drug loading and encapsulation efficiency}

The results of drug loading and encapsulation efficiencies with different $W_{\text {THF }} / W_{\text {PLGA }} / W_{\text {IBU }}$ ratios are shown in Table 1. The drug loading contents for the samples of $W_{\mathrm{THF}} / W_{\mathrm{PLGA}} /$ $W_{\mathrm{IBU}}=85 / 12.5 / 2.5, W_{\mathrm{THF}} / W_{\mathrm{PLGA}} / W_{\mathrm{IBU}}=85 / 11.25 / 3.75$, and $W_{\mathrm{THF}} / W_{\mathrm{PLGA}} / W_{\mathrm{IBU}}=85 / 10 / 5$ are $14.7 \% \pm 0.2 \%, 22 \% \pm 1.6 \%$ and $29.7 \% \pm 0.4 \%$, respectively. The values are a little smaller than the corresponding ones in theory $(16.7 \%, 25 \%$, and $33.3 \%$, respectively). As for the encapsulation efficiency, the samples exhibit high percentages (more than $88 \%$ ) and there are no remarkable differences among the different samples. These results reveal that our method is a simple and efficient way to prepare films with high drug loading and encapsulation efficiency. The high drug loading and encapsulation efficiency can be explained by the solubility of IBU and PLGA in THF, but their insolubility in water. THF, however, is intersoluble with water. When the mixed solution spreads on the water surface, the THF molecules immediately diffuse into the water phase, leaving the PLGA and IBU molecules to quickly solidify due to their indissolubility in water. Thus, high drug loading and encapsulation efficiency are obtained.

\section{Electron microscopy}

Figure 1 shows SEM images of the IBU-loaded PLGA film with the ratio of $W_{\text {THF }} / W_{\text {PLGA }} / W_{\text {IBU }}=85 / 12.5 / 2.5$.

Table I Drug loading and encapsulation efficiency (\%) at various IBU/PLGA weight ratios ( \pm SD, $n=3$ )

\begin{tabular}{llll}
\hline Ratio of $W_{\text {THF }} / W_{\text {PLGA }} / W_{\text {IBU }}$ & $\mathbf{8 5 / I 2 . 5 / 2 . 5}$ & $\mathbf{8 5} / \mathrm{II.25/3.75}$ & $\mathbf{8 5 / I 0 / 5}$ \\
\hline Drug loading (\%) & $14.7 \pm 0.2$ & $22.0 \pm 1.6$ & $29.7 \pm 0.4$ \\
Theoretical loading (\%) & 16.7 & 25 & 33.3 \\
Encapsulation efficiency (\%) & $88.2 \pm 1.2$ & $88.0 \pm 6.6$ & $89.1 \pm 1.3$ \\
\hline
\end{tabular}

Abbreviations: IBU, ibuprofen; PLGA, poly(lactic-co-glycolic acid).

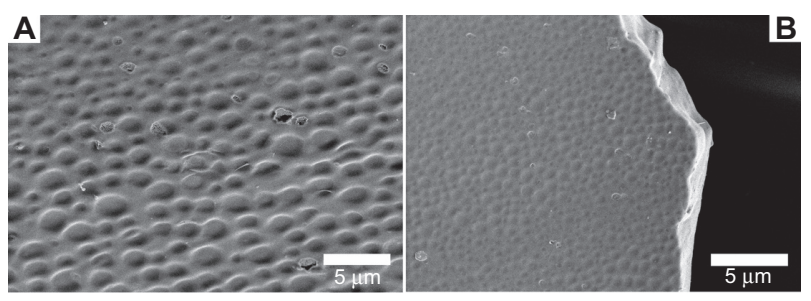

Figure I SEM images of IBU-loaded PLGA films ( $\left.\mathrm{W}_{\mathrm{THF}} / \mathrm{W}_{\mathrm{PLGA}} / \mathrm{W}_{\mathrm{IBU}}=85 / 12.5 / 2.5\right)$, the surface (A), and the thickness (B).

Abbreviations: IBU, ibuprofen; PLGA, poly(lactic-co-glycolic acid); SEM, scanning electron microscopy.

Figure 1A exhibits the top surface of the film, which has a convex pattern. The diameter of each convex shape is $1.52 \pm 0.45 \mu \mathrm{m}$. Figure 1B shows a cross-section image of the film, which has a smooth surface, indicating the absence of a void in the film.

Figure 2 shows the SEM images of films with different ratios of $W_{\mathrm{THF}} / W_{\mathrm{PLGA}} / W_{\mathrm{IBU}}$. Figure 2A shows the film with a ratio of $W_{\mathrm{THF}} / W_{\mathrm{PLGA}} / W_{\mathrm{IBU}}=85 / 11.25 / 3.75$. It shows that the film has a convex pattern on the top surface. The diameter of each convex shape is $0.87 \pm 0.11 \mu \mathrm{m}$. The insert in Figure 2A shows the rough cross-section surface which indicates the presence of voids in the film. Figure 2B shows the film with a ratio of $W_{\mathrm{THF}} / W_{\mathrm{PLGA}} / W_{\mathrm{IBU}}=85 / 10 / 5$. The top surface is relatively smooth and has no convex pattern. Furthermore, the cross-section of the film is rough, which indicates that there are voids in the film.

The SEM images in Figure 1 and Figure 2 show that there are voids in the film formed with higher drug concentration $\left(W_{\mathrm{THF}} / W_{\mathrm{PLGA}} / W_{\mathrm{IBU}}=85 / 11.25 / 3.75\right.$ and $\left.W_{\mathrm{THF}} / W_{\mathrm{PLGA}} / W_{\mathrm{IBU}}=85 / 10 / 5\right)$ while there are no obvious voids in the film formed at lower drug concentration $\left(W_{\mathrm{THF}} / W_{\mathrm{PLGA}} / W_{\mathrm{IBU}}=85 / 12.5 / 2.5\right)$. There is a convex pattern in the samples of $W_{\mathrm{THF}} / W_{\mathrm{PLGA}} / W_{\mathrm{IBU}}=85 / 12.5 / 2.5$ and $W_{\mathrm{THF}} /$ $W_{\mathrm{PLGA}} / W_{\mathrm{IBU}}=85 / 11.25 / 3.75$. However, there is no convex pattern for the sample of $W_{\mathrm{THF}} / W_{\mathrm{PLGA}} / W_{\mathrm{IBU}}=85 / 10 / 5$. The convex-patterned surface may result from the surface tension
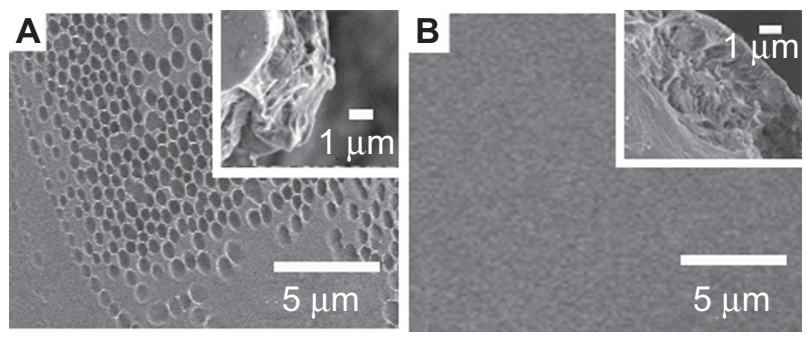

Figure 2 SEM images of IBU-loaded PLGA films, $W_{T H F} / W_{P L G A} / W_{1 B U}=85 / 11.25 / 3.75$ (A) and $W_{\text {THF }} / W_{\text {PLGA }} / W_{\text {IBU }}=85 / 10 / 5$ (B).

Abbreviations: IBU, ibuprofen; PLGA, poly(lactic-co-glycolic acid); SEM, scanning electron microscopy. 
when the phase separation proceeds at the top surface of the polymer solution, ie, the air/solution interface. ${ }^{24}$ The films have a dimension of about $33 \mathrm{~cm}^{2}$, which is as large as the Baker's dimension. The thickness of all the films with different weight ratios is in the range of 2 to $5 \mu \mathrm{m}$. In short, the ratio of $W_{\mathrm{THF}} / W_{\mathrm{PLGA}} / W_{\mathrm{IBU}}$ has a large influence on the surface morphology and structure of the films. For the formation of IBU-loaded PLGA film, the THF solvent diffuses quickly into water which leaves the undissolved IBU and PLGA solidified. In this case, the IBU and PLGA form a thin film on the surface of water. It is known that IBU and PLGA can dissolve well in THF as molecular state, and therefore the drug is distributed homogenously in the formed films.

\section{XRD measurements}

$\mathrm{X}$-ray diffraction (XRD) patterns were established on the pure IBU, pure PLGA, and the IBU-loaded PLGA films, in order to study the physical state of these systems. Generally, when the patterns of two crystal forms are identical in terms of peak positions, they have the same internal crystal structure, whereas if the patterns differ, the crystals have a different internal structure and are recognized as polymorphs. ${ }^{28}$ The XRD pattern of pure PLGA shown in Figure 3a has no evident brand, which denotes an amorphous polymeric compound. Figure $3 \mathrm{~b}$ shows the XRD patterns of pure IBU, which yields typical peaks at $16^{\circ}, 20^{\circ}$, and $22^{\circ}$, showing the crystalline substance. In the curves of IBU-loaded PLGA films shown in Figure $3 \mathrm{c}, \mathrm{d}$, and e, peaks at $16^{\circ}, 20^{\circ}$, and $22^{\circ}$ were detected in the three samples, which demonstrates that IBU had a crystalline state in the film. The difference in the relative

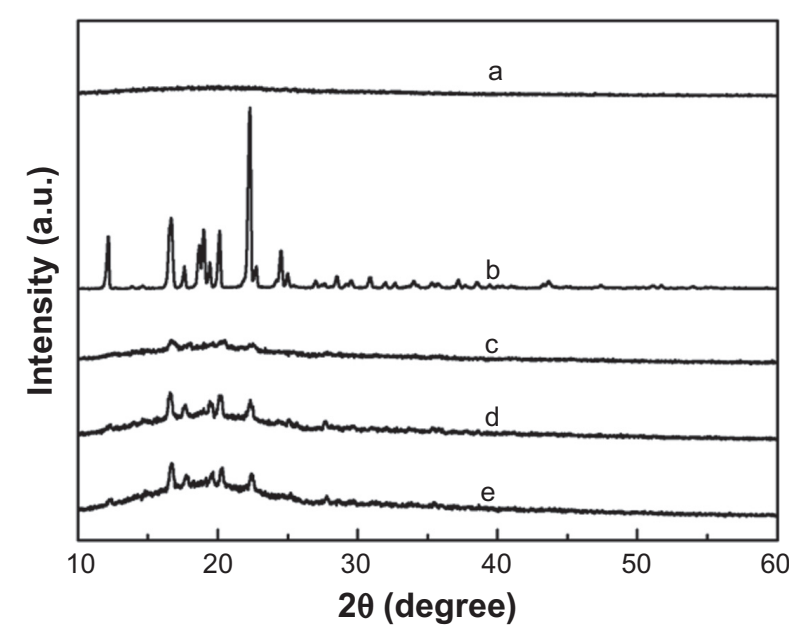

Figure $3 \times R D$ patterns of: a) pure IBU, b) PLGA, and IBU-loaded PLGA films: c) $W_{\text {THF }} / W_{\text {PLGA }} / W_{\text {IBU }}=85 / 12.5 / 2.5 ;$ d) $W_{\text {THF }} / W_{\text {PLGA }} / W_{\text {IBU }}=85 / 11.25 / 3.75$ and e) $W_{\text {THF }} /$ $W_{\text {PLGA }} / W_{\text {IBU }}=85 / 10 / 5$.

Abbreviations: IBU, ibuprofen; PLGA, poly(lactic-co-glycolic acid); XRD, X-ray diffraction. intensity of the peaks is due either to the variation of the crystal habit, because the relative abundance of the planes exposed to X-ray source is altered, or to differences in the size of the crystals. ${ }^{28}$

\section{DSC measurements}

In order to further study the drug-polymer interaction, the DSC thermal characteristics of IBU-loaded PLGA films (c, d, and e) are compared with pure PLGA (a) and pure IBU (b) in Figure 4. The endothermic peak of PLGA (curve a) is observed at $56^{\circ} \mathrm{C}$, which can be attributed to the glasstransition temperature of PLGA. The curve in Figure 4 shows a sharp endothermic peak at $78^{\circ} \mathrm{C}$, which indicates that the melting peak of pure IBU is $78^{\circ} \mathrm{C}$. The curves $\mathrm{c}, \mathrm{d}$, and $\mathrm{e}$ in Figure 4 show the peaks of different drug-loading films. Comparing with pure IBU, the melting points are 65,75 , and 73 for the samples of $W_{\mathrm{THF}} / W_{\mathrm{PLGA}} / W_{\mathrm{IBU}}=85 / 12.5 / 2.5, W_{\mathrm{THF}} /$ $W_{\mathrm{PLGA}} / W_{\mathrm{IBU}}=85 / 11.25 / 3.75$ and $W_{\mathrm{THF}} / W_{\mathrm{PLGA}} / W_{\mathrm{IBU}}=85 / 10 / 5$, respectively. The shift of the melting point indicates a decrease of drug crystallinity. These results are consistent with the XRD analysis, which reveals that IBU is crystalline while PLGA is amorphous in the drug-loaded film.

\section{In vitro release studies}

We examined the drug release behaviors of the as-prepared IBU-loaded PLGA films, in order to reveal their potential use in drug delivery systems. Figure 5 shows the release profiles of pure IBU and different ratios of $W_{\mathrm{THF}} / W_{\mathrm{PLGA}} / W_{\mathrm{IBU}}$ films in phosphate buffered solution (PBS, pH 6.8). Curve (a) shows that the pure IBU releases rapidly into the release medium

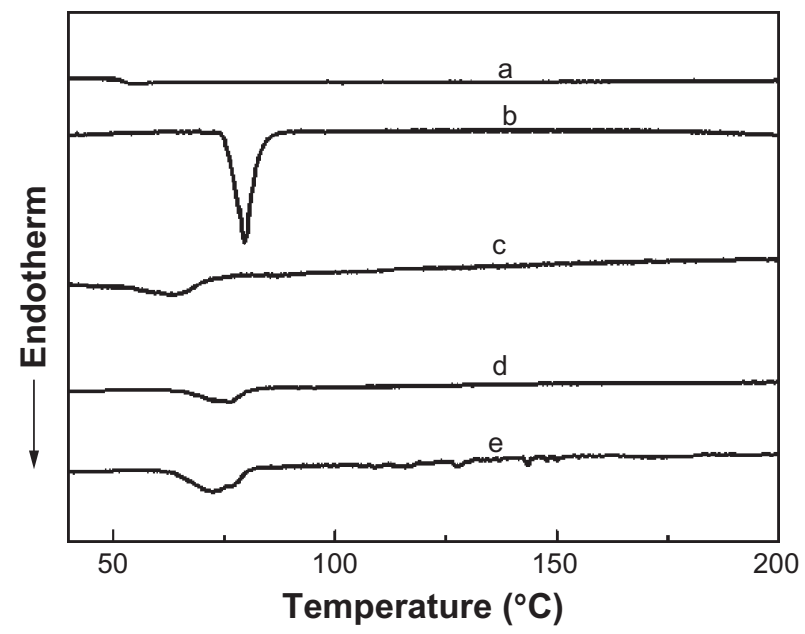

Figure 4 DSC curves of PLGA (a), pure IBU (b), and IBU-loaded films: c) $W_{\text {THF }}$ ' $W_{\text {PLGA }} / W_{\text {IBU }}=85 / / 2.5 / 2.5$; d) $W_{\text {THF }} / W_{\text {PLGA }} / W_{\text {IBU }}=85 / I 1.25 / 3.75$ and (e) $W_{\text {THF }} / W_{\text {PLGA }} /$ $W_{\mathrm{IBU}}=85 / 10 / 5$.

Abbreviations: DSC, differential scanning calorimetry; IBU, ibuprofen; PLGA, poly(lactic-co-glycolic acid). 


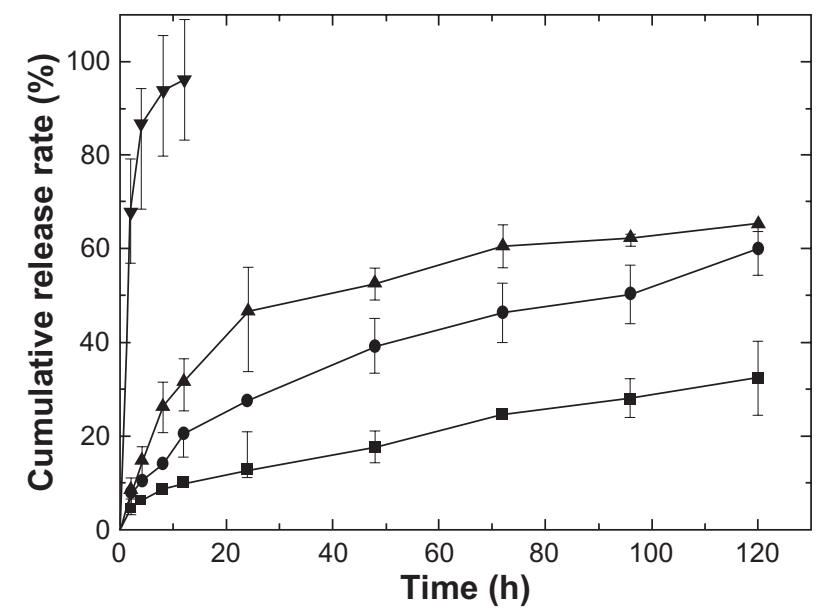

Figure 5 Cumulative release of different samples in $\mathrm{pH} 6.8$ phosphate buffered solution, films with ratios of $W_{\text {THF }} / W_{\text {PLGA }} / W_{\text {IBU }}=85 / I 2.5 / 2.5(-\square-), W_{\text {THF }} / W_{\text {PLGA }} /$

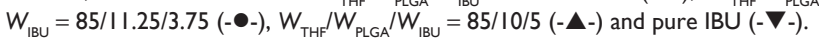
Abbreviation: IBU, ibuprofen.

with a release mount of nearly $100 \%$ in 12 hours. In contrast, in the sample with a ratio of $W_{\mathrm{THF}} / W_{\mathrm{PLGA}} / W_{\mathrm{IBU}}=85 / 12.5 / 2.5$, the release reached $12.7 \% \pm 1.5 \%$ within 24 hours, and reached $32.4 \% \pm 7.8 \%$ in 120 hours. In the sample with a ratio of $W_{\mathrm{THF}} / W_{\mathrm{PLGA}} / W_{\mathrm{IBU}}=85 / 11.25 / 3.75$, the release rate reached $27.5 \% \pm 6.4 \%$ in 24 hours and $60 \% \pm 5.5 \%$ in 120 hours. However, the release reached $46.6 \% \pm 9.5 \%$ in 24 hours and $65.3 \% \pm 1.6 \%$ in 120 hours in the sample with a ratio of $W_{\mathrm{THF}} / W_{\mathrm{PLGA}} / W_{\mathrm{IBU}}=85 / 10 / 5$. The different release percentages over the same time, show that the release rate decreases as the drug loading decreases. This can be due to the fewer drug molecules diffusing through the polymer network with the decrease of the drug loading. The polymer network becomes more porous as the drug concentration decreases, so that the soluble IBU molecules have to travel a longer distance from the pores to the release medium. The burst effect is not observed in the release, which can be explained as follows. On the one hand, the IBU is distributed homogeneously in the polymer without much surface segregation. On the other hand, rinsing the film decreases the amount of drug associated on the surface. These results show that a more controlled release can be obtained when changing the weight ratio of the drug, which indicates the potential application of the as-prepared drug-loaded PLGA products in a drug controlled delivery system.

In order to better understand the importance of the release mediums on the drug release kinetics from PLGA-based films, the other two release mediums were chosen to mimic the in vitro release. Figure 6 shows the release profiles of the sample with $W_{\mathrm{THF}} / W_{\mathrm{PLGA}} / W_{\mathrm{IBU}}=85 / 12.5 / 2.5$ from the release mediums, $\mathrm{HCl}$ aqueous solution $(\mathrm{pH} 1.2)$ and $\mathrm{PBS}(\mathrm{pH} 7.4)$.

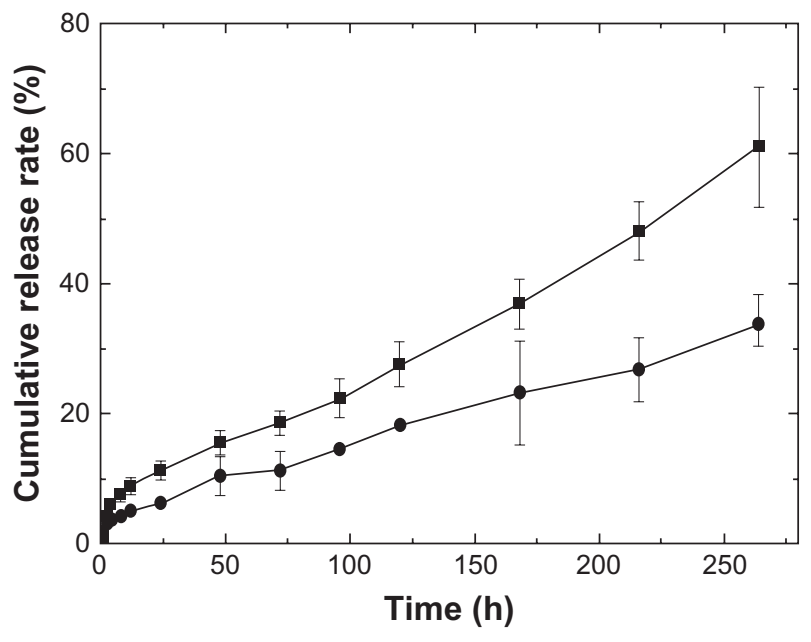

Figure 6 Cumulative release of the sample with $\mathrm{W}_{\mathrm{THF}} / \mathrm{W}_{\mathrm{PLGA}} / \mathrm{W}_{\mathrm{IBU}}=85 / 12.5 / 2.5$ in different release mediums, $\mathrm{pH} 7.4$ phosphate buffered solution (- -) and $\mathrm{pH} \mathrm{I.2} \mathrm{HCl}$ aqueous solution (-๑-).

The time to reach $30 \%$ of drug release in PBS was 135 hours compared with 238 hours in $\mathrm{HCl}$ aqueous solution, which reveals that the drug release kinetic is faster at high $\mathrm{pH}$ than at low $\mathrm{pH}$. The decreased release rate of IBU in acidic media ( $\mathrm{pH}$ 1.2) may have been due to the decreased solubility of IBU. The $\mathrm{p} K \mathrm{a}$ of IBU is known to be 4.42 and thus it has low aqueous solubility when the $\mathrm{pH}$ of the medium is below or close to 4.42 . What is more, matrix erosion which is affected by medium $\mathrm{pH}$ is also expected to affect the drug release. It is known that PLGA hydrolysis is equilibrium-limited in acidic environments, while it is not limited by equilibrium in basic conditions. Thus the erosion of the matrix increases when the $\mathrm{pH}$ of the release medium increases.

\section{Drug release kinetics}

The kinetics of IBU release from the films are determined by fitting the curves to distinct models, Zero order, First order, Higuchi release model, Ritger-Peppas, and Biexponential model. The equations for the different models and the regression coefficient $(R)$ are given in Table 2 and the best-fit model is selected on the basis of $R$.

For the sample $W_{\mathrm{THF}} / W_{\mathrm{PLGA}} / W_{\mathrm{IBU}}=85 / 12.5 / 2.5$ in $\mathrm{pH}$ 7.4 PBS and $W_{\mathrm{THF}} / W_{\mathrm{PLGA}} / W_{\mathrm{IBU}}=85 / 10 / 5$ in $\mathrm{pH}$ 6.8 PBS, we observe that $R$ value is larger when fitted to a biexponential equation compared with other equations, which indicates a biexponentia release from the PLGA-based films. The $R$ value is higher when the samples $\left(W_{\mathrm{THF}} / W_{\mathrm{PLGA}} / W_{\mathrm{IBU}}=85 / 12.5 / 2.5\right.$ and $\left.W_{\mathrm{THF}} / W_{\mathrm{PLGA}} / W_{\mathrm{IBU}}=85 / 11.25 / 3.75\right)$ are fitted to the RitgerPeppas kinetics equation in the $\mathrm{pH} 6.8$ release medium, which indicates a Ritger-Peppas release from the PLGA matrix. When the sample $\left(W_{\mathrm{THF}} / W_{\mathrm{PLGA}} / W_{\mathrm{IBU}}=85 / 12.5 / 2.5\right)$ 
Table 2 The equations for the different models and the regression coefficient $(R)$ of IBU-loaded PLGA films in vitro

\begin{tabular}{|c|c|c|c|}
\hline Samples & Model & Equation & $\boldsymbol{R}$ \\
\hline \multirow{6}{*}{$\mathrm{W}_{\mathrm{THF}} / \mathrm{W}_{\mathrm{PLGA}} / \mathrm{W}_{\mathrm{IBU}}=85 / 12.5 / 2.5, \mathrm{pH}=1.2$} & Zero-order kinetics & $Q=0.1201 t+3.0237$ & 0.9972 \\
\hline & First-order kinetics & $\ln (100-Q)=-0.0014 t+4.5786$ & 0.9970 \\
\hline & Higuchi & $Q=1.6027 t^{1 / 2}-1.3078$ & 0.9085 \\
\hline & Ritger-Peppas & $\ln Q=0.4062 \ln t+0.6594$ & 0.9528 \\
\hline & Biexponential & $100-Q=97.839 \mathrm{e}^{-0.1052 t}+104 \mathrm{e}^{-0.139 t}$ & $R_{\alpha}=0.9340$ \\
\hline & & & $R_{\beta}=0.9690$ \\
\hline \multirow{6}{*}{$W_{\text {THF }} / W_{P L G A} / W_{\text {IBU }}=85 / / 2.5 / 2.5, p H=7.4$} & Zero-order kinetics & $Q=0.2057 t+4.2613$ & 0.9956 \\
\hline & First-order kinetics & $\ln (100-Q)=-0.003 t+4.5832$ & 0.9830 \\
\hline & Higuchi & $Q=3.2695 t^{1 / 2}-3.0312$ & 0.9654 \\
\hline & Ritger-Peppas & $\ln Q=0.5008 \ln t+0.9880$ & 0.9894 \\
\hline & Biexponential & $100-Q=98.283 \mathrm{e}^{-1.0574 t}+94.796 \mathrm{e}^{-0.2002 t}$ & $R_{\alpha}=0.9834$ \\
\hline & & & $R_{\beta}=0.9957$ \\
\hline \multirow{6}{*}{$W_{T H F} / W_{P L G A} / W_{\text {IBU }}=85 / / 2.5 / 2.5, p H=6.8$} & Zero-order kinetics & $Q=0.2281 t+6.3468$ & 0.9920 \\
\hline & First-order kinetics & $\ln (100-Q)=-0.0028 t+4.5497$ & 0.9953 \\
\hline & Higuchi & $Q=2.8396 t^{1 / 2}+0.1087$ & 0.9948 \\
\hline & Ritger-Peppas & $\ln Q=0.4659 \ln t+1.1722$ & 0.9955 \\
\hline & Biexponential & $100-Q=96.181 \mathrm{e}^{-0.5508 t}+91.87 \mathrm{e}^{-0.2075 t}$ & $R_{\alpha}=0.9857$ \\
\hline & & & $R_{\beta}=0.9945$ \\
\hline \multirow[t]{6}{*}{$W_{\text {THF }} / W_{\text {PLGA }} / W_{\text {IBU }}=85 / I 1.25 / 3.75, p H=6.8$} & Zero-order kinetics & $Q=0.4216 t+12.675$ & 0.9736 \\
\hline & First-order kinetics & $\ln (100-Q)=-0.0067 t+4.4868$ & 0.9896 \\
\hline & Higuchi equation & $Q=5.3614 t^{1 / 2}+0.5101$ & 0.9953 \\
\hline & Ritger-Peppas & $\ln Q=0.4993 \ln t+1.6995$ & 0.9973 \\
\hline & Biexponential & $100-Q=93.85 \mathrm{e}^{-1.0107 t}+80.762 \mathrm{e}^{-0.3462 t}$ & $R_{\alpha}=0.9933$ \\
\hline & & & $R_{\beta}=0.9862$ \\
\hline \multirow{6}{*}{$W_{T H F} / W_{P L G A} / W_{\text {IBU }}=85 / 10 / 5, p H=6.8$} & Zero-order kinetic & $Q=0.235 t+28.738$ & 0.9369 \\
\hline & First-order kinetics & $\ln (100-Q)=-0.0052 t+4.2746$ & 0.9761 \\
\hline & Higuchi equation & $Q=4.4906 t^{1 / 2}+13.555$ & 0.9801 \\
\hline & Ritger-Peppas & $\ln Q=0.4194 \ln t+2.1991$ & 0.9826 \\
\hline & Biexponential & $100-Q=90.346 \mathrm{e}^{-1.52 \mathrm{t}}+50.022 \mathrm{e}^{-0.1150 \mathrm{t}}$ & $\mathrm{R}_{\alpha}=0.9968$ \\
\hline & & & $\mathrm{R}_{\beta}=0.9945$ \\
\hline \multirow[t]{2}{*}{ Pure IBU, $\mathrm{pH}=6.8$} & Zero-order kinetics & $Q=6.4129 t+35.624$ & 0.7701 \\
\hline & First-order kinetics & $\ln (100-Q)=-0.2586 t+4.1051$ & 0.9510 \\
\hline
\end{tabular}

Note: Where $Q$ is the fraction of drug release, $t$ is the time, $R$ is the regression coefficient.

is released from $\mathrm{pH} 1.2$ release medium, it fits to Zero-order kinetics. As for the release of pure IBU, the $R$ value is larger when fitting to First-order kinetics than Zero-order kinetics. Drug release from matrices is usually complex and though some processes may be clearly classified as either diffusion or erosion controlled, drug release is mostly governed by both mechanisms. However, the above results may provide a better understanding of the drug controlled release from the drug-loaded films.

\section{Conclusion}

In summary, a very simple, basic method was developed to fabricate drug-loaded biocompatible polymeric films by directly spreading polymer/drug solution on the nonsolvent surface. PLGA and IBU were used as the model polymer and drug, respectively. By controlling the weight ratio of
IBU and PLGA, different drug loading percentage films can be prepared. The drug release behaviors of the as-prepared products show their potential applications in a drug controlled delivery system. The release rate of IBU from these films into phosphate buffered solutions appeared to depend on a number of factors including drug loading content and the $\mathrm{pH}$ of the release mediums. This method can easily be scaled up and potentially extended to the fabrication of other drug-loaded composites for the application in drug delivery systems.

\section{Acknowledgments}

This work is supported by the National Natural Science Foundation of China (NSFC, No. 20803044, No. 20803043), and the Excellent Young Scientist Foundation of Shandong Province (BS2009CL002). 


\section{Disclosure}

The authors declare no conflicts of interest.

\section{References}

1. Perugini P, Genta I, Conti B, et al. Periodontal delivery of ipriflavone: new chitosan/PLGA film delivery system for a lipophilic drug. Int $J$ Pharm. 2003;252:1-9.

2. Ye M, Kim S, Park K. Issues in long-term protein delivery using biodegradable microparticles. J Contr Release. 2010;146:241-260.

3. Schade A, Niwa T, Takeuchi H, et al. Aqueous colloidal polymer dispersions of biodegradable DL-lactide/glycolide copolymer as basis for latex films: a new approach for the development of biodegradable depot systems. Int J Pharm. 1995;117:209-217.

4. Siegel SJ, Kahn JB, Metzger K, et al. Effect of drug type on the degradation rate of PLGA matrices. Eur J Pharm Biopharm. 2006;64: 287-293.

5. Klose D, Siepmann F, Willart JF, et al. Drug release from PLGA-based microparticles: effects of the "microparticle:bulk fluid" ratio. Int JPharm. 2010;383:123-131.

6. Xu Q, Czernuszka JT. Controlled release of amoxicillin from hydroxyapatite-coated poly (lactic-co-glycolic acid) microspheres. J Contr Release. 2008;127:146-153.

7. Fernández-Carballido A, Herrero-Vanrell R, Molina-Martinez IT, et al. Biodegradable ibuprofen-loaded PLGA microspheres for intraarticular administration effect of Labrafil addition on release in vitro. Int J Pharm. 2004;279:33-41.

8. Schliecker G, Schmidt C, Fuchs S, et al. Hydrolytic degradation of poly(lactide-co-glycolide) films: effect of oligomers on degradation rate and crystallinity. Int J Pharm. 2003;266:39-49.

9. Wang J, Wang BM, Schwendeman SP. Characterization of the initial burst release of a model peptide from poly(D,L-lactide-co-glycolide) microspheres. J Contr Release. 2002;82:289-307.

10. Acharya G, Shin CS, Vedantham K, et al. A study of drug release from homogeneous PLGA microstructures. J Contr Release. 2010;146 201-206.

11. Jackson JK, Smith J, Letchford K, et al. Characterization of perivascular poly (lactic-co-glycolic acid) films containing paclitaxel. Int J Pharm. 2004;283:97-109.

12. Dorta MJ, Oliva A, Munguia O, et al. In-vivo release of fluoropyrimidines from PLGA film implants. J Pharm Pharmacol. 2002;54:757-763.

13. Dorta MJ, Santovena A, Llabres M, et al. Potential applications of PLGA film-implants in modulating in vitro drugs release. Int J Pharm. 2002; 24:149-156.
14. Ma D, McHugh AJ. The interplay of membrane formation and drug release in solution-cast films of polylactide polymers. Int J Pharm. 2010;388:1-12.

15. Knill CJ, Mistry J, Smart G, Groocock MR, Williams HJ, Kennedy JF. Alginate fibres modified with unhydrolysed and hydrolysed chitosans for wound dressings. Carbohyd Polym. 2004;55:65-76.

16. Shi SA, Wang XH, Guo G, Fan M, Huang MJ, Qian ZY. Preparation and characterization of microporous poly(D,L-lactic acid) film for tissue engineering scaffold. Int J Nanomed. 2010;5:1049-1055.

17. Jiang BB, Li BY. Tunable drug loading and release from polypeptide multilayer nanofilms. Int J Nanomed. 2009;4:37-53.

18. Patel NA, Patel NJ, Patel RP. Design and evaluation of transdermal drug delivery system for curcumin as an anti-inflammatory drug. Drug Dev Ind Pharm. 2009;35:234-242.

19. Moneghini M, Bellich B, Baxa P, et al. Microwave generated solid dispersions containing Ibuprofen. Int J Pharm. 2008;361:125.

20. Pang J, Luan Y, Li F, et al. Ionic liquid-assisted synthesis of silica particles and their application in drug release. Mater Lett. 2010;64: 2509-2512.

21. Newa M, Bhandari KH, Li DX, et al. Preparation, characterization and in vivo evaluation of ibuprofen binary solid dispersions with poloxamer 188. Int J Pharm. 2007;343:228-237.

22. Jain JA. The manufacturing techniques of various drug loaded biodegradable poly (lactide-co-glycolide) (PLGA) devices. Biomaterials. 2000;21:2475-2490.

23. Wang Y, Liu Z, Han B, et al. A simple route to micropatterned polymer surfaces. Chem Commun. 2004;800-801.

24. Tang Y, Singh J. Controlled delivery of aspirin: effect of aspirin on polymer degradation and in vitro release from PLGA based phase sensitive systems. Int J Pharm. 2008;357:119-125.

25. Zhou S, Deng X, Li X, Jia W, Liu L. Synthesis and characterization of biodegradable low molecular weight aliphatic polyesters and their use in protein-delivery systems. J Appl Polym Sci. 2004;91:1848-1856.

26. Ferronato K, Bruxel F, Araujo FA, Teixeira HF, Koester LS. Use of the dialysis bag method to evaluate the in vitro release of drugs from submicrometric carriers. Lat Am J Pharm. 2010;29:313-320.

27. Luo YF, Chen DW, Ren LX, Zhao XL, Qin J. Solid lipid nanoparticles for enhancing vinpocetine's oral bioavailability. J Contr Release. 2006; 114:53-59.

28. Garekani HA, Sadeghi F, Badiee A, Mostafa SA, Rajabi-Siahboomi AR. Crystal habit modifications of ibuprofen and their physicomechanical characteristics. Drug Dev Ind Pharm. 2001;27:803-809.
International Journal of Nanomedicine

\section{Publish your work in this journal}

The International Journal of Nanomedicine is an international, peerreviewed journal focusing on the application of nanotechnology in diagnostics, therapeutics, and drug delivery systems throughout the biomedical field. This journal is indexed on PubMed Central, MedLine, CAS, SciSearch $®$, Current Contents ${ } /$ Clinical Medicine,

\section{Dovepress}

Journal Citation Reports/Science Edition, EMBase, Scopus and the Elsevier Bibliographic databases. The manuscript management system is completely online and includes a very quick and fair peer-review system, which is all easy to use. Visit http://www.dovepress.com/ testimonials.php to read real quotes from published authors. 